\title{
OCCUPATIONAL EXPOSURE TO ASBESTOS IS ASSOCIATED WITH INCREASED MORTALITY IN MEN RECRUITED FOR A POPULATION-BASED STUDY IN GERMANY
}

\author{
KATJA REPP ${ }^{1}$, ROBERTO LORBEER ${ }^{1}$, TILL ITTERMANN ${ }^{1}$, SVEN GLÄSER ${ }^{2}$, ULRICH JOHN, \\ WOLFGANG HOFFMANN ${ }^{1}$, and HENRY VÖLZKE ${ }^{1}$
}

\author{
Ernst Moritz Arndt University of Greifswald, Greifswald, Germany \\ ${ }^{1}$ Institute for Community Medicine \\ ${ }^{2}$ Department of Internal Medicine B - Cardiology, Intensive Care, Pulmonary Medicine and Infectious Diseases \\ ${ }^{3}$ Institute of Epidemiology and Social Medicine
}

\begin{abstract}
Objectives: Occupational exposure to asbestos is associated with increased mortality which, however, has not been thoroughly validated in a general population. We have aimed at exploring whether this association may be confirmed within a population-based setting after adjustment for confounders. Furthermore, the impact of tobacco consumption on the association between occupational exposure to asbestos and mortality is assessed. Material and Methods: We used data from 2072 (224 exposed) male participants of the Study of Health in Pomerania. Information on exposure to asbestos is based on a selfreport. Median follow-up time was 11.3 years. All-cause mortality and cause-specific mortality of exposed and non-exposed men were compared using mortality rate ratios, Kaplan-Meier analyses and multivariable Cox regression. Results: During the follow-up, $52(23.2 \%)$ exposed and $320(17.3 \%)$ non-exposed participants deceased. Exposed subjects had increased hazard ratios (HR) for all-cause mortality ( $\mathrm{HR}=1.48,95 \% \mathrm{CI}$ : 1.1-2), benign lung disease mortality (HR=3, 95\% CI: 1.187.62) and stomach cancer mortality $(\mathrm{HR}=4.59,95 \% \mathrm{CI}: 1.53-13.76)$. The duration of exposure (per 10 years) was associated with all-cause $(\mathrm{HR}=1.21,95 \% \mathrm{CI}$ : $1.07-1.36)$ and benign lung disease mortality (HR=1.68, 95\% CI: 1.26-2.22). Smokers occupationally exposed to asbestos had the highest risk for all-cause $(\mathrm{HR}=3.70,95 \% \mathrm{CI}$ : $2.19-6.27)$ and cancer mortality $(\mathrm{HR}=4.56,95 \% \mathrm{CI}$ : 1.99-10.48) as compared to non-asbestos exposed non-smokers. Conclusion: Our results confirm associations of occupational exposure to asbestos with all-cause, benign lung disease, and stomach cancer mortality and underline the impact of joint effects of asbestos and smoking on mortality.
\end{abstract}

Key words:

Occupational exposure to asbestos, Mortality, Cancer, Population based, Epidemiology, Asbestos

\footnotetext{
This research work was funded by grants from the German Federal Ministry for Education and Research, from the Ministry for Education, Research and Cultural Affairs and the Ministry for Social Affairs of the State Mecklenburg-West Pomerania. Furthermore, this work is a part of the research project Greifswald Approach to Individualized Medicine (GANI_MED). Grantors: German Federal Ministry of Education and Research and Ministry of Cultural Affairs of the German Federal State of Mecklenburg - West Pomerania, grant number: 03IS2061A. Funding was additionally related to German Asthma and COPD Network (COSYCONET); Grant sponsor: BMBF, grant number: 01GI0883. Projects manager: Prof. dr. Ralf Ewert.

Received: May 28, 2013. Accepted: December 18, 2014.

Corresponding author: K. Repp, University Medicine Greifswald, Institute for Community Medicine, 48 Walther Rathenau, D-17475 Greifswald, Germany (e-mail: reppkatja@gmail.com).
} 


\section{INTRODUCTION}

Though banned in many countries because of its toxic effects, an estimated number of 125 million people worldwide are still occupationally exposed to asbestos [1]. The exposure to asbestos is not without consequence: inhaled fibers persist to some extent in the lungs, resulting in acute and chronic inflammation, leading to fibrosis and malignant neoplasms [2]. Asbestos causes asbestosis and mesothelioma as well as cancer of the lung, larynx [1,3], and ovaries [4]. Non-population-based cohort studies observed elevated all-cause mortality, benign lung disease mortality and cancer mortality among subjects who were occupationally exposed to asbestos [5-7]. According to estimates by the World Health Organization (WHO) in 2010, more than 107000 persons die each year from asbestosrelated lung cancer, mesothelioma and asbestosis [1].

Despite extensive research in this field, some questions remain unanswered. There are conflicting results on whether asbestos may be responsible for further, extra-pulmonary diseases. Several studies suggest that asbestos exposure increases the risk for mortality from extra-pulmonary neoplasms [8-10] whereas others did not find such associations [8]. Furthermore, non-population-based cohort studies showed that the joint effects of smoking and asbestos led to an increased risk for lung cancer mortality in smokers who were occupationally exposed to asbestos [11-14]. No studies, however, have specifically investigated the impact of possible joint effects between tobacco smoking and exposure to asbestos on all-cause mortality or cancer mortality in the general population.

Further limitations of previous studies include the lack of an adequate control group and insufficient information on potential confounders. Several previous studies [5,7,15] used standardized mortality ratios to compare the mortality of asbestos workers with the mortality of the general population. Since national or regional mortality registers usually do not provide information on socioeconomic status and smoking habits, adjusting for these confounders was not possible in most studies. Thus some uncertainty remains whether the observed association between occupational exposure to asbestos and mortality may have been substantially influenced by confounding or joint effects with cigarette smoking.

This population-based study, conducted in West Pomerania, Northeast Germany, provides a well-characterized population of asbestos exposed and non-exposed subjects, including information on socioeconomic status and smoking habits. The use of asbestos in Eastern Germany strongly increased in the 1960's and remained on a high level until the German reunification in 1990. Between 1970 and 1990, around $50000 \mathrm{t}$ of asbestos were imported annually and subsequently processed in eastern German factories [16]. The imported asbestos consisted predominantly of chrysotile; amphiboles were only imported for acid protection products [16]. Asbestos was finally banned in reunited Germany in 1993. The continuous occupational exposure to asbestos until 1990 predisposes the Northeast German population for investigating the effects of occupational exposure to asbestos on mortality in a population-based approach.

We have aimed at exploring whether our populationbased study confirms the previously observed association of occupational exposure to asbestos with all-cause and specific-cause mortality. Furthermore, we have sought to investigate if this population-based approach may add new evidence for these associations when considering confounding. Specifically, we have addressed the role of smoking in the association of occupational exposure to asbestos with all-cause and cancer mortality.

\section{MATERIAL AND METHODS}

\section{Study population}

The Study of Health in Pomerania (SHIP) is a populationbased study in West Pomerania, a region in the north-east of Germany [17]. A stratified random sample from the population aged 20-79 years old was drawn. The sample was 
selected using population registries, where all German citizens have to be registered. Only individuals with German citizenship and main residency in the study area were included. The net sample (without migrated or deceased persons) comprised 6267 eligible subjects, 4308 (68.8\%) of whom agreed to participate in the SHIP. Baseline data was collected between October 1997 and May 2001. All participants gave informed written consents. The study protocol is consistent with the principles of the Declaration of Helsinki and was approved by the Ethics Committee of the University of Greifswald.

\section{Assessments}

Presence and duration of occupational exposure to asbestos, information on smoking habits, and socio-economic status were assessed using standardized computer-assisted personal interviews. Participants were asked 2 questions regarding their occupational exposure to asbestos:

- Have you ever been exposed to asbestos in your professional life for at least 6 months (for example when producing or using asbestos containing textiles, brake pads, sealing products, asbestos containing construction materials or asbestos demolition work)?

\section{- If so, for how many years have you been exposed?}

Smoking status was assessed accounting for cigarette, cigar and pipe smoking; participants were categorized as never smokers, former smokers, and current smokers. Former smokers were those who agreed to have ever smoked but denied to smoke currently. To investigate the impact of smoking status on the association between occupational exposure to asbestos and mortality we defined a 6-category variable with combined information on asbestos exposure and smoking status. The educational level was divided into 4 categories ( $<10$ years, $10-11$ years, $12-14$ years or $>14$ years of required education). Household income was divided by the square root of household members to calculate the equivalent household income [18]. Occupations were coded according to the system of the International
Labour Association and converted into the International Social Index of Occupational Status (ISEI) [19]. Height and weight were measured, and the body mass index (BMI) was calculated (weight $(\mathrm{kg}) /$ height $^{2}\left(\mathrm{~m}^{2}\right)$ ). Systolic and diastolic blood pressure was measured using a digital blood pressure monitor (HEM-705CP, Omron Corporation, Tokyo, Japan). Serum low-density lipoprotein (LDL) cholesterol was measured photometrically (Hitachi 704, Roche, Mannheim, Germany). Glycated hemoglobin $\left(\mathrm{HbA}_{1 \mathrm{c}}\right)$ was measured by means of high pressure liquid chromatography with photometric detection (Diamat analyzer; BioRad, Munich, Germany).

\section{Exclusion criteria}

Since only 29 (1.3\%) out of 2192 female participants had been occupationally exposed to asbestos, we limited our analyses to male participants. Out of the male participants we excluded 36 subjects because assessment of occupational asbestos exposure was not possible. Furthermore, we excluded 8 men because of missing values in confounding variables. This resulted in a final study population of 2072 men.

\section{Mortality-follow-up}

Information on vital status was collected from population registries at annual intervals. Subjects were censored at loss to follow-up. The period between the baseline examination and the date of vital status collection or the date of death was considered as follow-up length. The median time of follow-up was 11.3 years (25th percentile: 10.3 years, 75 th percentile: 11.8 years, 21.721 person-years).

Death certificates were requested from the local health authorities and coded by a certified nosologist according to the International Classification of Diseases, 10th revision (ICD-10). Two specialists for internal medicine independently validated the underlying cause of death and performed a joint reading with a 3rd internist in cases of disagreement. 


\section{Statistical analyses}

Continuous data is expressed as median and interquartile range, and categorical data is expressed in terms of absolute numbers and percent values. Participants were divided into 2 groups according to the presence or absence of former occupational asbestos exposure. Comparisons between the 2 groups were made using Mann-Whitney's U-test (continuous data) and $\mathrm{Chi}^{2}$ test (categorical data). The relative risks for all-cause and specific-cause mortality were calculated as mortality rate ratio. Excess mortality assuming causality was calculated as mortality rate differences. Cumulative survival was calculated using KaplanMeier analysis. Survival curves were compared using the log-rank test.

We performed the multivariable Cox proportional hazards regression analysis to associate exposure to asbestos and duration of occupational exposure to asbestos with mortality. The proportional hazard assumption for the Cox regression models was checked by visual inspection of Schoenfeld residuals and was not violated. Multivariable fractional polynomials were used to test for nonlinear associations between the confounders and mortality; the regression models were adapted in the case of non-linearity. Hazard ratios (HR) for allcause mortality and specific causes of death were calculated; values have been given with their $95 \%$ confidence intervals $(\mathrm{CI})$.

Three different regression models were applied: the 1st one adjusted for age, the 2nd one additionally adjusted for educational level, and the final one adjusted for age, educational level and smoking status. We proceeded to conduct the following sensitivity analyses: In the 1st step we adjusted the final regression model additionally for ISEI and household income. From this analysis we excluded 177 men because of missing values in ISEI or household income. In the 2nd step we replaced smoking status in the final regression model by the estimation of the lifetime smoking exposure:

$$
\begin{gathered}
\text { pack years }(\mathrm{PY})=\text { number of cigarettes per day } / 20 \times \\
\text { number of years of smoking }
\end{gathered}
$$

To investigate the impact of the lifetime smoking exposure on the association between occupational exposure to asbestos and mortality we defined a 6-category variable with combined information on asbestos exposure and PY. From this part of the analyses we excluded 342 males because the estimation of PY was not possible: 294 former or current smokers who smoked only occasionally (fewer than 1 cigarette per day), 38 former or current smokers who smoked exclusively cigars or pipes but did not smoke cigarettes, 10 former cigarette smokers with missing values in PY.

All statistical analyses were performed using Stata 11.0 (Stata Corporation, College Station, TX, USA). A $p<0.05$ was considered statistically significant.

\section{RESULTS}

\section{Baseline characteristics}

Among the study population of 2072 men, 224 reported a previous occupational exposure to asbestos (10.8\%). There were no significant differences in terms of age, blood pressure, metabolic markers (LDL, $\mathrm{HbA}_{1 \mathrm{C}}$ ), smoking status, and household income between the 2 groups. Educational level and occupational status were significantly lower, lifetime smoking exposure (PY) was significantly higher, follow-up time was significantly shorter in asbestos-exposed men as compared to non-exposed men. Median age of death in asbestos-exposed men was 72.3 years whereas age of death in non-exposed men was 76.6 years (Table 1).

\section{Mortality rate ratios and mortality rate difference}

During the follow-up, 52 exposed and 320 non-exposed subjects died from all causes (Table 2).

Men who had been occupationally exposed to asbestos had significantly higher mortality rates for all-cause mortality, stomach cancer mortality and mortality due to malignancies of the gastrointestinal tract as compared 
Table 1. Baseline characteristics for male participants with and without occupational exposure to asbestos

\begin{tabular}{|c|c|c|c|}
\hline \multirow[b]{2}{*}{ Variable } & \multicolumn{2}{|c|}{ Occupational exposure to asbestos } & \multirow[b]{2}{*}{$\mathrm{p}$} \\
\hline & $\begin{array}{c}\text { yes } \\
(\mathrm{N}=224)\end{array}$ & $\begin{array}{c}\text { no } \\
(\mathrm{N}=1848)\end{array}$ & \\
\hline Follow-up time [years] (Me (25-75th percentile)) & $11.1(9.9-11.7)$ & $11.3(10.3-11.8)$ & 0.047 \\
\hline Age at baseline [years] (Me (25-75th percentile)) & $53.0(40.0-62.0)$ & $51.0(37.0-65.0)$ & 0.411 \\
\hline Age at time of death [years] (Me (25-75th percentile)) & $72.3(62.6-78.8)$ & $76.6(69.2-82.3)$ & 0.006 \\
\hline Body mass index $\left[\mathrm{kg} / \mathrm{m}^{2}\right](\mathrm{Me}(25-75$ th percentile $))$ & $27.2(25.4-30.0)$ & $27.4(24.8-30.0)$ & 0.778 \\
\hline \multicolumn{4}{|l|}{ Blood pressure [mm Hg] ( $\mathrm{Me}$ (25-75th percentile)) } \\
\hline systolic & $141.5(130.0-154.0)$ & $141.0(129.0-153.0)$ & 0.588 \\
\hline diastolic & $85.8(80.3-93.8)$ & $85.0(78.0-93.0)$ & 0.182 \\
\hline $\mathrm{HbA}_{1 \mathrm{c}}[\%](\mathrm{Me}(25-75$ th percentile $))$ & $5.4(5.0-5.9)$ & $5.4(5.0-5.9)$ & 0.342 \\
\hline LDL [mmol/l] (Me (25-75th percentile)) & $3.6(2.7-4.3)$ & $3.6(2.9-4.3)$ & 0.840 \\
\hline \multicolumn{4}{|l|}{ Smoking status $[\mathrm{n}(\%)]$} \\
\hline never smokers & $35.0(15.6)$ & $359.0(19.4)$ & \\
\hline former smokers & $106.0(47.3)$ & $840.0(45.5)$ & 0.390 \\
\hline current smokers & $83.0(37.1)$ & $649.0(35.1)$ & \\
\hline Pack years $^{\mathrm{a}}(\mathrm{Me}$ (25-75th percentile)) & $16.5(5.4-31.0)$ & $11.7(1.5-26.0)$ & 0.001 \\
\hline \multicolumn{4}{|l|}{ Educational level [n (\%)] } \\
\hline$\leq 9$ years & $18.0(8.0)$ & $115.0(6.2)$ & \\
\hline $10-11$ years & $95.0(42.4)$ & $656.0(35.5)$ & 0.001 \\
\hline $12-14$ years & $89.0(39.7)$ & $680.0(36.8)$ & \\
\hline$\geq 15$ years & $22.0(9.8)$ & $397.0(21.5)$ & \\
\hline $\begin{array}{l}\text { Equivalent household income }{ }^{\mathrm{b}} \text { [euro] (Me (25-75th } \\
\text { percentile)) }\end{array}$ & $949.0(677.9-1175.0)$ & $959.4(677.9-1342.1)$ & 0.082 \\
\hline $\begin{array}{l}\text { International Socio-Economic Index of Occupational } \\
\text { Status (ISEI) (Me (25-75th percentile)) }\end{array}$ & $35.0(31.0-41.0)$ & $39.0(32.0-57.0)$ & $<0.001$ \\
\hline
\end{tabular}

Me - median; $\mathrm{HbA}_{\mathrm{lc}}$ - glycated hemoglobin; LDL - low density lipoprotein.

${ }^{a}$ Pack years - the number of cigarettes per day $/ 20 \times$ number of years of smoking.

${ }^{\mathrm{b}}$ Income (euro) $/ \sqrt{\text { the number of household members. }}$

${ }^{c}$ Chi ${ }^{2}$ test (nominal data) or Mann-Whitney U-test (interval data) were performed.

to non-exposed men. We observed no cases of death due to asbestosis or malignancies of the small intestine in the entire study population and no cases of death due to cancer of the larynx or cancer of the esophagus in asbestos exposed subjects. We observed 1 case of death due to mesothelioma in a man without self-reported occupational exposure to asbestos. Mortality rate differences representing excess mortality are presented in the Table 3.

\section{Multivariable Cox regression analyses}

The following results refer to the final model (adjusted for age, smoking status and educational level). Compared to men who were not occupationally exposed to asbestos, exposed subjects showed significantly increased risk for allcause mortality, benign lung disease mortality, mortality due to malignancies of the gastrointestinal tract in general and stomach cancer mortality in particular (Table 4). 
Table 2. Mortality rate ratio for subjects with/without occupational exposure to asbestos

\begin{tabular}{|c|c|c|c|c|c|}
\hline \multirow{2}{*}{ Cause of death } & \multirow{2}{*}{ Measuring unit } & \multicolumn{2}{|c|}{ Exposure to asbestos } & \multicolumn{2}{|c|}{$\begin{array}{l}\text { Mortality ratio } \\
{[\mathrm{RR}(95 \% \mathrm{CI})]}\end{array}$} \\
\hline & & $\begin{array}{c}\text { yes } \\
(\mathrm{N}=224)\end{array}$ & $\begin{array}{c}\text { no } \\
(\mathrm{N}=1848)\end{array}$ & crude & age adjusted ${ }^{1}$ \\
\hline Total & $\begin{array}{l}\text { cumulative survival time [years] } \\
\text { deceased } \\
\text { mortality rate (per } 1000 \text { person years) }\end{array}$ & $\begin{array}{r}2255.1 \\
52.0 \\
23.1\end{array}$ & $\begin{array}{r}19466.7 \\
320.0 \\
16.4\end{array}$ & $1.40(1.03-1.89)^{*}$ & $1.55(1.17-2.06)^{*}$ \\
\hline $\begin{array}{l}\text { Diseases of the circulatory } \\
\text { system (I00-I99) }\end{array}$ & $\begin{array}{l}\text { deceased } \\
\text { mortality rate (per } 1000 \text { person years) }\end{array}$ & $\begin{array}{r}10.0 \\
4.4\end{array}$ & $\begin{array}{r}110.0 \\
5.6\end{array}$ & $0.79(0.37-1.50)$ & $0.92(0.48-1.74)$ \\
\hline $\begin{array}{l}\text { Benign lung disease } \\
\text { (J00-J99) }\end{array}$ & $\begin{array}{l}\text { deceased } \\
\text { mortality rate (per } 1000 \text { person years) }\end{array}$ & $\begin{array}{l}6.0 \\
2.7\end{array}$ & $\begin{array}{r}20.0 \\
1.0\end{array}$ & $2.59(0.85-6.69)$ & $3.31(1.33-8.20)^{*}$ \\
\hline asbestosis (J61, J92) & deceased & 0.0 & 0.0 & & \\
\hline $\begin{array}{l}\text { Malignant neoplasm } \\
\quad(\text { C00-C99) }\end{array}$ & $\begin{array}{l}\text { deceased } \\
\text { mortality rate (per } 1000 \text { person years) }\end{array}$ & $\begin{array}{r}19.0 \\
8.4\end{array}$ & $\begin{array}{r}117.0 \\
6.0\end{array}$ & $1.40(0.81-2.29)$ & $1.51(0.93-2.45)$ \\
\hline $\begin{array}{l}\text { malignant neoplasm } \\
\text { of the respiratory system } \\
(\mathrm{C} 30-\mathrm{C} 34)\end{array}$ & $\begin{array}{l}\text { deceased } \\
\text { mortality rate (per } 1000 \text { person years) }\end{array}$ & $\begin{array}{l}7.0 \\
3.1\end{array}$ & $\begin{array}{r}31.0 \\
1.6\end{array}$ & $1.95(0.72-4.51)$ & $2.11(0.93-4.80)$ \\
\hline lung cancer (C34) & $\begin{array}{l}\text { deceased } \\
\text { mortality rate (per } 1000 \text { person years) }\end{array}$ & $\begin{array}{l}7.0 \\
3.1\end{array}$ & $\begin{array}{r}28.0 \\
1.4\end{array}$ & $2.16(0.80-5.06)$ & $2.32(1.01-5.33)^{*}$ \\
\hline larynx cancer (C32) & $\begin{array}{l}\text { deceased } \\
\text { mortality rate (per } 1000 \text { person years) }\end{array}$ & $\begin{array}{l}0.0 \\
0.0\end{array}$ & $\begin{array}{l}3.0 \\
0.2\end{array}$ & & \\
\hline Mesothelioma (C45) & $\begin{array}{l}\text { deceased } \\
\text { mortality rate (per } 1000 \text { person years) }\end{array}$ & $\begin{array}{l}0.0 \\
0.0\end{array}$ & $\begin{array}{l}1.0 \\
0.1\end{array}$ & & \\
\hline $\begin{array}{l}\text { Malignant neoplasm } \\
\text { of digestive organs } \\
\text { (C15-C27) }\end{array}$ & $\begin{array}{l}\text { deceased } \\
\text { mortality rate (per } 1000 \text { person years) }\end{array}$ & $\begin{array}{l}9.0 \\
4.0\end{array}$ & $\begin{array}{r}46.0 \\
2.4\end{array}$ & $1.69(0.73-3.49)$ & $1.79(0.88-3.65)$ \\
\hline $\begin{array}{l}\text { malignant neoplasm of the } \\
\text { digestive tract (C15-C21) }\end{array}$ & $\begin{array}{l}\text { deceased } \\
\text { mortality rate (per } 1000 \text { person years) }\end{array}$ & $\begin{array}{l}8.0 \\
3.5\end{array}$ & $\begin{array}{r}27.0 \\
1.4\end{array}$ & $2.56(1.00-5.79)^{*}$ & $2.68(1.23-5.85)^{*}$ \\
\hline stomach cancer $(\mathrm{C} 16)$ & $\begin{array}{l}\text { deceased } \\
\text { mortality rate (per } 1000 \text { person years) }\end{array}$ & $\begin{array}{l}5.0 \\
2.2\end{array}$ & $\begin{array}{r}10.0 \\
0.5\end{array}$ & $4.32(1.16-13.86)^{*}$ & $4.49(1.54-13.08)^{*}$ \\
\hline $\begin{array}{l}\text { colorectal cancer } \\
(\mathrm{C} 18-\mathrm{C} 20)\end{array}$ & $\begin{array}{l}\text { deceased } \\
\text { mortality rate (per } 1000 \text { person years) }\end{array}$ & $\begin{array}{l}3.0 \\
1.3\end{array}$ & $\begin{array}{r}14.0 \\
0.7\end{array}$ & $1.85(0.34-6.63)$ & $1.97(0.59-6.62)$ \\
\hline
\end{tabular}

$\mathrm{RR}$ - rate ratio; $\mathrm{CI}$ - confidence interval.

${ }^{1}$ Based on Poisson regression models adjusted for age.

$* \mathrm{p}<0.05$.

We observed no significant association of occupational exposure to asbestos with total cancer mortality or lung cancer mortality. Occupational exposure to asbestos was not associated with cardiovascular mortality.

\section{Duration of exposure}

Duration of occupational exposure to asbestos was associated with a significantly increased risk for all-cause mortality and benign lung disease mortality (Table 4). 
Table 3. Mortality rate differences of expected deaths based on crude mortality rates of participants without occupational exposure to asbestos vs. occurred deaths in participants with occupational exposure to asbestos

\begin{tabular}{lcccc}
\hline \multirow{2}{*}{\multicolumn{1}{c}{ Cause of death }} & \multicolumn{2}{c}{$\begin{array}{c}\text { Deaths } \\
{[\mathrm{n}]}\end{array}$} & \multicolumn{2}{c}{ Mortality rate difference } \\
\cline { 2 - 5 } & expected & occurred & in total & $\begin{array}{c}\text { per 1000 person } \\
\text { years }\end{array}$ \\
\cline { 2 - 5 } Total & 37.0 & 52 & 15.0 & 6.7 \\
Diseases of the circulatory system & 12.6 & 10 & -2.6 & -1.2 \\
Benign lung disease & 2.3 & 6 & 3.7 & 1.7 \\
Malignant neoplasm & 13.5 & 19 & 5.5 & 2.4 \\
Malignant neoplasm of the respiratory system & 3.6 & 7 & 3.4 & 1.5 \\
Lung cancer & 3.2 & 7 & 3.8 & 1.7 \\
Larynx cancer & 0.5 & 0 & -0.5 & -0.2 \\
Mesothelioma & 0.2 & 0 & -0.2 & -0.1 \\
Malignant neoplasm of digestive organs & 5.4 & 9 & 3.6 & 1.6 \\
Malignant neoplasm of the digestive tract & 3.2 & 8 & 4.8 & 2.1 \\
Stomach cancer & 1.1 & 5 & 3.9 & 1.7 \\
Colorectal cancer & 1.6 & 3 & 1.4 & 0.6 \\
\hline
\end{tabular}

Additionally we observed a borderline significant association between duration of exposure and lung cancer mortality. Duration of exposure was not significantly associated with other mortality causes.
We repeated all multivariable analyses additionally adjusted for ISEI and income. Finally, we performed all multivariable analyses adjusting for PY instead of smoking status. These sensitivity analyses did not change the results substantially.

Table 4. Hazard ratios for participants with/without occupational exposure to asbestos and for duration of occupational exposure to asbestos

\begin{tabular}{|c|c|c|c|c|}
\hline Cause of death & Exposure & $\begin{array}{c}\text { Model } 1 \\
{[\mathrm{HR}(95 \% \mathrm{CI})]}\end{array}$ & $\begin{array}{c}\text { Model } 2 \\
{[\mathrm{HR}(95 \% \mathrm{CI})]}\end{array}$ & $\begin{array}{c}\text { Model } 3 \\
{[\mathrm{HR}(95 \% \mathrm{CI})]}\end{array}$ \\
\hline Total & $\begin{array}{l}\text { yes/no } \\
\text { duration of exposure (per } 10 \text { years) }\end{array}$ & $\begin{array}{l}1.58(1.18-2.12)^{*} \\
1.24(1.10-1.39)^{*}\end{array}$ & $\begin{array}{l}1.52(1.13-2.05)^{*} \\
1.22(1.08-1.38)^{*}\end{array}$ & $\begin{array}{l}1.48(1.10-2.00)^{*} \\
1.21(1.07-1.36)^{*}\end{array}$ \\
\hline $\begin{array}{l}\text { Diseases of the circulatory } \\
\text { system (ICD I00-I99) }\end{array}$ & $\begin{array}{l}\text { yes/no } \\
\text { duration of exposure (per } 10 \text { years) }\end{array}$ & $\begin{array}{l}0.94(0.49-1.80) \\
0.97(0.72-1.31)\end{array}$ & $\begin{array}{l}0.92(0.47-1.75) \\
0.96(0.71-1.31)\end{array}$ & $\begin{array}{l}0.90(0.47-1.73) \\
0.96(0.70-1.30)\end{array}$ \\
\hline $\begin{array}{l}\text { Benign lung diseases } \\
\text { (ICD J00-J99) }\end{array}$ & $\begin{array}{l}\text { yes/no } \\
\text { duration of exposure (per } 10 \text { years) }\end{array}$ & $\begin{array}{l}3.46(1.38-8.67)^{*} \\
1.82(1.38-2.40)^{*}\end{array}$ & $\begin{array}{l}3.03(1.20-7.68)^{*} \\
1.70(1.28-2.26)^{*}\end{array}$ & $\begin{array}{l}3.00(1.18-7.62)^{*} \\
1.68(1.26-2.22)^{*}\end{array}$ \\
\hline $\begin{array}{l}\text { Malignant neoplasm } \\
\text { (ICD C00-C99) }\end{array}$ & $\begin{array}{l}\text { yes/no } \\
\text { duration of exposure (per } 10 \text { years) }\end{array}$ & $\begin{array}{l}1.45(0.89-2.35) \\
1.15(0.93-1.42)\end{array}$ & $\begin{array}{l}1.39(0.85-2.27) \\
1.14(0.92-1.40)\end{array}$ & $\begin{array}{l}1.31(0.80-2.14) \\
1.12(0.90-1.38)\end{array}$ \\
\hline $\begin{array}{l}\text { respiratory neoplasm } \\
(\mathrm{C} 30-\mathrm{C} 34)\end{array}$ & $\begin{array}{l}\text { yes/no } \\
\text { duration of exposure (per } 10 \text { years) }\end{array}$ & $\begin{array}{l}2.00(0.88-4.57) \\
1.40(1.05-1.86)^{*}\end{array}$ & $\begin{array}{l}1.72(0.75-3.94) \\
1.35(1.02-1.82)^{*}\end{array}$ & $\begin{array}{l}1.42(0.62-3.29) \\
1.30(0.97-1.74)\end{array}$ \\
\hline $\begin{array}{l}\text { lung cancer } \\
\text { (ICD C34) }\end{array}$ & $\begin{array}{l}\text { yes/no } \\
\text { duration of exposure (per } 10 \text { years) }\end{array}$ & $\begin{array}{l}2.17(0.94-4.99) \\
1.43(1.07-1.90)^{*}\end{array}$ & $\begin{array}{l}1.83(0.79-4.21) \\
1.38(1.03-1.84)^{*}\end{array}$ & $\begin{array}{l}1.49(0.64-3.47) \\
1.32(0.98-1.78)\end{array}$ \\
\hline
\end{tabular}


Table 4. Hazard ratios for participants with/without occupational exposure to asbestos and for duration of occupational exposure to asbestos - cont.

\begin{tabular}{lllll}
\hline \multicolumn{1}{c}{ Cause of death } & \multicolumn{1}{c}{ Exposure } & \multicolumn{1}{c}{$\begin{array}{c}\text { Model 1 } \\
\text { [HR }(95 \% \text { CI })]\end{array}$} & $\begin{array}{c}\text { Model 2 } \\
{[\text { HR }(95 \% \text { CI })]}\end{array}$ & $\begin{array}{c}\text { Model 3 } \\
\text { [HR (95\% CI)] }\end{array}$ \\
\hline $\begin{array}{l}\text { Neoplasm of digestive organ } \\
\text { (C15-C27) }\end{array}$ & $\begin{array}{l}\text { yes/no } \\
\text { duration of exposure (per 10 years) }\end{array}$ & $1.70(0.83-3.49)$ & $1.70(0.83-3.50)$ & $1.63(0.79-3.37)$ \\
neoplasm of the digestive & yes/no & $2.56(1.16-5.65)^{*}$ & $2.69(1.20-6.00)^{*}$ & $2.65(1.19-5.95)^{*}$ \\
tract (C15-C21) & duration of exposure (per 10 years) & $1.13(0.73-1.73)$ & $1.16(0.75-1.79)$ & $1.16(0.75-1.79)$ \\
stomach cancer & yes/no & $4.47(1.52-13.11)^{*}$ & $4.61(1.54-13.77)^{*}$ & $4.59(1.53-13.76)^{*}$ \\
(ICD C16) & duration of exposure (per 10 years) & $1.37(0.82-2.29)$ & $1.37(0.81-2.30)$ & $1.36(0.81-2.28)$ \\
colorectal cancer & yes/no & $1.97(0.56-6.88)$ & $2.24(0.63-8.00)$ & $2.32(0.65-8.34)$ \\
(C18-C20) & duration of exposure (per 10 years) & $0.99(0.46-2.14)$ & $1.09(0.50-2.37)$ & $1.09(0.50-2.41)$ \\
\hline
\end{tabular}

ICD - International Statistical Classification of Diseases and Related Health Problems.

Cox proportional hazards analysis: model 1 - adjusted for age; model 2 - adjusted for age, educational level; model 3 - adjusted for age, educational level and smoking status.

HR - hazard ratio; $\mathrm{CI}$ - confidence interval.

$* \mathrm{p}<0.05$.

Table 5. Joint effects of smoking status and occupational asbestos exposure

\begin{tabular}{lcc}
\hline \multicolumn{1}{c}{ Participant } & \multicolumn{2}{c}{$\begin{array}{c}\text { Mortality } \\
\text { [HR (95\% CI)] }\end{array}$} \\
\cline { 2 - 3 } & \multicolumn{2}{c}{ total } \\
\hline No asbestos, never smoker $(\mathrm{N}=359)$ & 1.00 (reference) & 1.00 (reference) \\
Asbestos, never smoker $(\mathrm{N}=35)$ & $0.89(0.27-2.87)$ & $0.90(0.12-6.86)$ \\
No asbestos, former smoker $(\mathrm{N}=840)$ & $1.14(0.81-1.60)$ & $1.18(0.65-2.16)$ \\
Asbestos, former smoker $(\mathrm{N}=106)$ & $1.66(1.01-2.70)^{*}$ & $1.44(0.60-3.49)$ \\
No asbestos, current smoker $(\mathrm{N}=649)$ & $2.21(1.52-3.21)^{*}$ & $3.08(1.65-5.74)^{*}$ \\
Asbestos, current smoker $(\mathrm{N}=83)$ & $3.70(2.19-6.27)^{*}$ & $4.56(1.99-10.48)^{*}$ \\
\hline
\end{tabular}

Cox proportional hazards analysis, model adjusted for age and educational level.

Cancer mortality refers to mortality due to malignant neoplasm (ICD C00-C99).

Abbreviations as in Table 2 and 4.

$* \mathrm{p}<0.05$.

Table 6. Joint effects between lifetime smoking exposure (pack years) and occupational asbestos exposure

\begin{tabular}{lcc}
\hline \multirow{2}{*}{ Participants } & \multicolumn{2}{c}{ Mortality } \\
& \multicolumn{2}{c}{$[\mathrm{HR}(95 \% \mathrm{CI})]$} \\
\cline { 2 - 3 } & total & cancer \\
\hline No asbestos, never smoker $(\mathrm{N}=359)$ & 1.00 (reference) & 1.00 (reference) \\
Asbestos, never smoker $(\mathrm{N}=35)$ & $0.86(0.27-2.79)$ & $0.87(0.11-6.65)$ \\
\hline
\end{tabular}


Table 6. Joint effects between lifetime smoking exposure (pack years) and occupational asbestos exposure - cont.

\begin{tabular}{|c|c|c|}
\hline \multirow[t]{2}{*}{ Participants } & \multicolumn{2}{|c|}{$\begin{array}{c}\text { Mortality } \\
{[\mathrm{HR}(95 \% \mathrm{CI})]}\end{array}$} \\
\hline & total & cancer \\
\hline \multicolumn{3}{|l|}{ No asbestos, smoker } \\
\hline$<20 \mathrm{PY}(\mathrm{N}=633)$ & $0.92(0.62-1.37)$ & $0.99(0.50-1.99)$ \\
\hline$\geq 20 \mathrm{PY}(\mathrm{N}=550)$ & $1.75(1.23-2.48)^{*}$ & $2.34(1.29-4.23)^{*}$ \\
\hline \multicolumn{3}{|l|}{ Asbestos, smoker } \\
\hline$<20 \mathrm{PY}(\mathrm{N}=66)$ & $1.54(0.77-3.09)$ & $1.44(0.40-5.08)$ \\
\hline$\geq 20 \mathrm{PY}(\mathrm{N}=87)$ & $2.95(1.85-4.72)^{*}$ & $3.31(1.53-7.17)^{*}$ \\
\hline
\end{tabular}

PY - pack year.

Other abbreviations as in Table 2 and 4.

$* \mathrm{p}<0.05$.

Cox proportional hazards analysis, model adjusted for age and educational level.

Cancer mortality refers to mortality due to malignant neoplasm (ICD C00-C99).
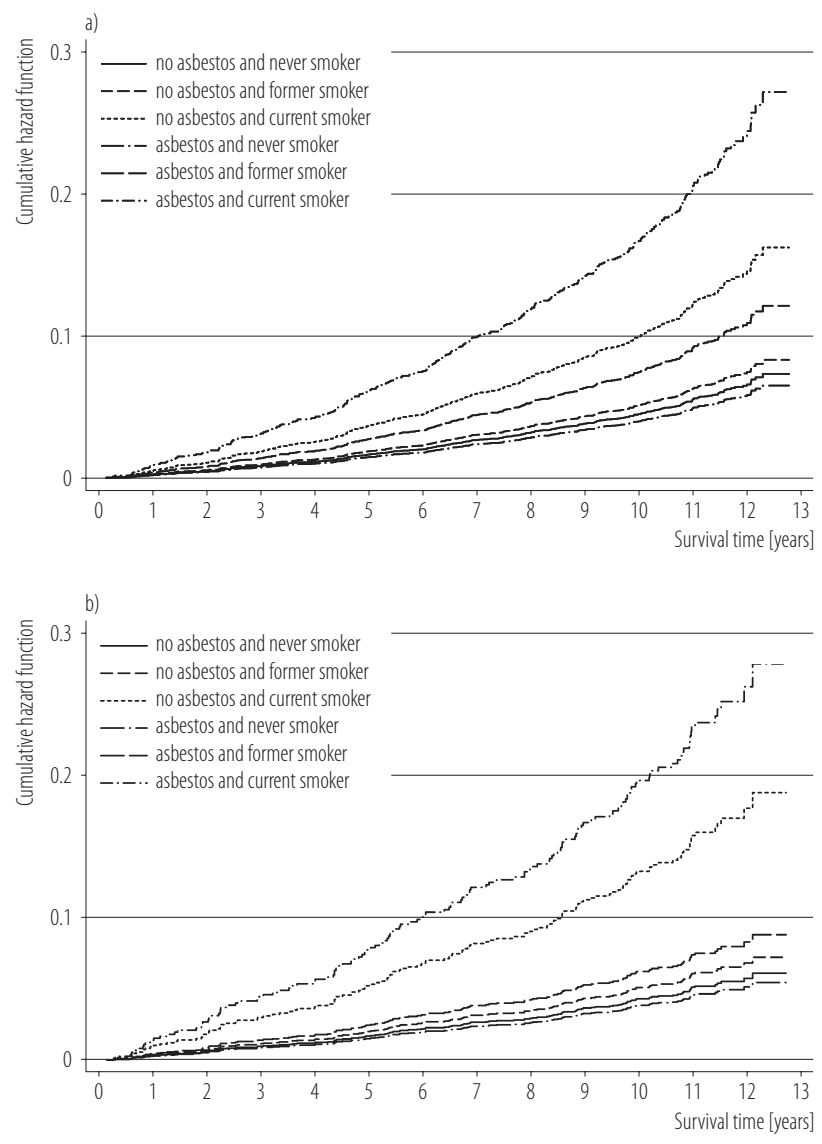

Fig. 1. Impact of the joint effects of occupational exposure to asbestos and smoking status on a) all-cause mortality and b) on cancer mortality

\section{The role of smoking}

The Cox regression model, adjusted for age and educational level, did not show an increased risk for all-cause or cancer mortality in asbestos-exposed never smokers as compared to never smokers without asbestos exposure (Table 5, Figure 1). Participants who were current smokers at baseline had a significantly increased risk for all-cause and cancer mortality. The effect sizes were stronger in current smokers who were additionally exposed to asbestos as compared to current smokers who were not exposed to asbestos. Former smokers who were occupationally exposed to asbestos also had a significantly increased risk for all-cause mortality whereas non-exposed former smokers had no significantly increased risk for all-cause mortality. Results of the sensitivity analyses using categories of lifetime smoking exposure instead of smoking status did not change the results substantially (Table 6).

\section{DISCUSSION}

Our study demonstrates that former occupational exposure to asbestos affects mortality risks and causes of death in the general population of West Pomerania. Overall, our results regarding all-cause and specific-cause mortality 
support findings from previously conducted non-population-based cohort studies. Deaths from benign lung diseases, lung cancer and stomach cancer seem to contribute most notably to excess mortality in participants with occupational exposure to asbestos. Tobacco consumption increases the mortality risk in men who were occupationally exposed to asbestos.

\section{All-cause mortality and respiratory mortality}

As in SHIP, significantly increased risks for all-cause mortality [6,9,20,21] and benign lung disease mortality $[7,22,23]$ had previously been observed. The significant association of the duration of occupational exposure to asbestos with all-cause mortality and benign lung disease mortality, respectively, underline the validity of our findings. Although we did not observe a statistically significant association between occupational exposure to asbestos and mortality from all cancers or lung cancer, in particular we observed positive non-significant trends that were in line with previous findings [9,15,21,23]. A borderline significant association of the duration of occupational exposure to asbestos with lung cancer mortality may support the assumption of causality between exposure to asbestos dust and lung cancer.

We did not observe any cases of death from mesothelioma or asbestosis among men occupationally exposed to asbestos. This is within the expected range since less than 1 case of death from mesothelioma or asbestosis was expected when considering the incidence of both diseases in Germany [24,25]. However, we observed 1 case of mesothelioma in a man without self-reported occupational exposure to asbestos whose working history (musician) does not suggest exposure to asbestos dust in his professional life. This may be a random finding, since more than $25 \%$ of mesothelioma in German men occur in the absence of occupational exposure to asbestos and may have been attributed to asbestos exposure in leisure time [26].

\section{Mortality due to gastrointestinal malignancies}

We observed significantly elevated mortality due to malignancies of the gastrointestinal tract in general and stomach cancer in particular among asbestos-exposed subjects as compared to non-exposed subjects. Although the missing association of duration of occupational exposure to asbestos with mortality from gastrointestinal malignancies and the relatively small number of cases limit the validity of this finding, an association of occupational exposure to asbestos with gastrointestinal malignancies is plausible.

Inhaled asbestos fibers are partly transported out of the lungs by mucociliary clearance and subsequently swallowed [2]. In addition, an inadvertent, direct ingestion of asbestos fibers is possible [8]. In vitro and in vivo studies showed that asbestos fibers penetrated and interacted not only with the mucosal cells of the pulmonary system but also with the mucosal cells of the gastrointestinal tract [27].

However, evidence regarding the carcinogenic potency of asbestos in the gastrointestinal tract as well as evidence regarding increased mortality from gastrointestinal malignancies in asbestos exposed subjects is limited and inconsistent [8]. Associations of occupational exposure to asbestos with increased mortality risk due to esophageal cancer [28], cancer of the small intestine [28] or cancer of the large intestine [29] have only been detected in single cohort studies. Increased mortality from stomach cancer in asbestos exposed subjects has previously been observed in several cohort studies [9,30-32].

\section{The role of smoking}

There are no previous studies investigating the impact of joint effects between occupational exposure to asbestos and smoking on all-cause or all-cancer mortality in the general population.

In our study, asbestos-exposed men who were active smokers at baseline showed the highest all-cause and 
all-cancer mortality. In contrast to active smokers without and former smokers with occupational exposure to asbestos who had significantly elevated all-cause mortality, former smokers without occupational exposure to asbestos had only a slightly, non-significantly elevated mortality risk. We did not observe increased mortality among asbestos-exposed never-smokers but since this group only comprised 35 men an association could have been left undetected due to low statistical power.

Overall, these results indicate that the association of occupational exposure to asbestos with all-cause and all-cancer mortality is strongly influenced by smoking status at baseline. Results of the sensitivity analyses using categories of lifetime smoking exposure instead of smoking status underline the impact of joint effects between smoking and asbestos on mortality risk and the importance of smoking prevention in persons with a history of occupational exposure to asbestos.

\section{Latency period of asbestos-related diseases}

Latency of asbestos related diseases is reported to range between 15 and more than 40 years after the 1st exposure [24,33,34]. Starting between 1997 and 2001 and ending in 2012 our follow-up was conducted in the midst of the epidemic of asbestos related diseases in Germany, which was provoked by excessive use of asbestos between 1955 and 1980 (West Germany) e.g., 1965-1990 (East Germany) [35]. The peak of asbestos related diseases in Germany is expected within the next years and future follow-up analyses of this cohort will hopefully give more insight into the association of occupational exposure to asbestos and mortality causes; especially malignancies of the airways and the gastrointestinal tract.

Unfortunately, we do not have information on the age at the 1st exposure and thus could not gain specific information on the latency period of asbestos related diseases in our study sample.

\section{Methodological strengths and weaknesses}

A distinct advantage of a population-based cohort design in general and this study in particular serves the possibility to assess detailed information on potential confounders including socioeconomic factors and smoking - for both asbestos exposed and non-exposed subjects. Our analyses revealed that the adjustment for the selected confounders reduced the observed effects of occupational exposure to asbestos on mortality but did not completely explain the association.

Notwithstanding the strengths of our study, the following drawbacks have to be taken into account when interpreting the presented results. First, although occupational exposure to asbestos was not a rare condition in Northeast Germany, the fraction of exposed men was clearly smaller as compared to the fraction of non-exposed men, which limited the statistical power. Second, the validity of findings on cause-specific mortality is limited by the small numbers of deceased men in each category. Third, we did not have sufficient information to investigate the impact of a possible healthy worker effect on the outcomes.

Fourth, the information on smoking describes smoking status and lifetime smoking exposure at the time of the baseline examination. Since no information on smoking habits during the time of occupational exposure to asbestos was available for this analysis, an analysis of time-sequence was not possible. Fifth, we did not have information on the exact circumstances of occupational exposure to asbestos, for example, the industry, within the framework of which the exposure took place, or the use of protective measures; therefore assessment of fiber years was not possible. The cumulative duration of exposure may only represent a rough estimate of the lifetime accumulated exposure. Additionally we have no information on the type of asbestos the participants of this study were exposed to. Although the predominant part of asbestos used in East Germany was chrysotile asbestos and amphiboles were hardly used in 
East Germany, some participants might have been exposed to amphiboles that might be responsible for some of the observed effects.

Sixth, the information on occupational exposure to asbestos was based on a self-report, which may have given rise to misclassification bias as a person might not have been aware of his or her exposure. It was not possible to investigate the extent of possible misclassification since $75 \%$ of the persons registered by the German Democratic Republic (GDR) authorities as "occupationally exposed to asbestos" did not agree to undergo voluntary, centrally organized medical examinations after the German reunification. These persons have not been further followed up; information on vital status, residency or morbidity is not available.

In this context we would like to report that the selfreported exposure to asbestos in our study was 5.9\% ( $10.8 \%$ in men), which is higher than expected when considering data on exposure provided by Eastern German authorities in 1990. At this time, around 122000 persons ( $0.8 \%$ of the East-German population older than 16$)$ were registered as "occupationally exposed to asbestos" [36]. When considering the fact that asbestos intensive construction and shipping industry were the most notable industries of the study area, the extent of exposure reported by the volunteers of our study seems to be plausible.

Finally, we would like to emphasize that this study only considers exposure to asbestos dusts that took place in the regular working environment. We did not have information on environmental exposure to asbestos dusts or exposure that arose during leisure time when conducting "do it yourself activities" like repairing houses and boats or constructing sheds using asbestos containing materials. This means that this study only considers exposure to asbestos dust that took place in the regular working environment and most likely underestimates the effects of asbestos dust on mortality in general.

\section{CONCLUSIONS}

This population-based cohort study does confirm significantly increased risks for all-cause mortality and benign lung disease mortality in men occupationally exposed to asbestos. The increased mortality due to gastrointestinal cancers in general and stomach cancer in particular may indicate that the carcinogenic potency of asbestos is not limited to the airways and mesothelium. Tobacco consumption significantly contributes to the association between occupational exposure to asbestos and mortality.

\section{ACKNOWLEDGMENTS}

The authors would like to thank Prof. Henri Wallaschofski and Prof. Marcus Dörr for the validation of the underlying mortality causes.

\section{REFERENCES}

1. World Health Organization. Geneva: WHO; 2014 [cited 2014 Dec 5]. Asbestos: Elimination of asbestos-related diseases. Fact sheet No. 343. Available from: http://www.who. int/mediacentre/factsheets/fs343/en/index.html.

2. Mossman BT, Lippmann M, Hesterberg TW, Kelsey KT, Barchowsky A, Bonner JC. Pulmonary endpoints (lung carcinomas and asbestosis) following inhalation exposure to asbestos. J Toxicol Environ Health B Crit Rev. 2011;14(1-4): 76-121, http://dx.doi.org/10.1080/10937404.2011.556047.

3. Hauptverband der Gewerblichen Berufsgenossenschaften (HVBG). [Occupational diseases caused by asbestos - development and prognosis]. Sankt Augustin; 2003 [cited 2014 Dec 5]. p. 15-23. Available from: http://publikationen.dguv.de/dguv/udt_dguv_main.aspx?FDOCUID= 24596. German.

4. Camargo MC, Stayner LT, Straif K, Reina M, Al-Alem U, Demers PA, et al. Occupational exposure to asbestos and ovarian cancer: A meta-analyses. Environ Health Perspect. 2011;119:1211-7, http://dx.doi.org/10.1289/ehp.1 003283. 
5. Pira E, Pelucchi C, Buffoni L, Palmas A, Turbiglio M, Negri $\mathrm{E}$, et al. Cancer mortality in a cohort of asbestos textile workers. Br J Cancer. 2005;92(3):580-6, http://dx.doi. org/10.1038/sj.bjc.6602240.

6. Bertolotti M, Ferrante D, Mirabelli D, Botta M, Nonnato M, Todesco A, et al. Mortality in the cohort of the asbestos cement workers in the Eternit plant in Casale Monferrato (Italy). Epidemiol Prev. 2008;32(4-5):218-28.

7. Hein MJ, Stayner LT, Lehman E, Dement JM. Follow-up study of chrysotile textile workers: Cohort mortality and exposure-response. Occup Environ Med. 2007;64(9):616-25, http://dx.doi.org/10.1136/oem.2006.031005.

8. Bunderson-Schelvan M, Pfau JC, Crouch R, Holian A. Nonpulmonary outcomes of asbestos exposure. J Toxicol Environ Health B Crit Rev. 2011;14(1-4):122-52, http:/dx. doi.org/10.1080/10937404.2011.556048.

9. Harding AH, Darnton A, Wegerdt J, McElvenny D. Mortality among British asbestos workers undergoing regular medical examinations (1971-2005). Occup Environ Med. 2009;66(7):487-95, http://dx.doi.org/10.1136/ oem.2008.043414.

10. Berry G, Newhouse ML, Wagner JC. Mortality from all cancers of asbestos factory workers in east London 193380. Occup Environ Med. 2000;57(11):782-5, http://dx.doi. org/10.1136/oem.57.11.782.

11. Yano E, Wang X, Wang M, Qiu H, Wang Z. Lung cancer mortality from exposure to chrysotile asbestos and smoking: A case-control study within a cohort in China. Occup Environ Med. 2010;67(12):867-71, http://dx.doi.org/10.11 36/oem.2009.051615.

12. Frost G, Darnton A, Harding AH. The effect of smoking on the risk of lung cancer mortality for asbestos workers in Great Britain (1971-2005). Ann Occup Hyg. 2011;55(3): 239-47, http://dx.doi.org/10.1093/annhyg/meq089.

13. Berry G, Liddell FD. The interaction of asbestos and smoking in lung cancer: A modified measure of effect. Ann Occup Hyg. 2004;48(5):459-62, http://dx.doi.org/10.1093/annhyg/ meh023.
14. Liddell FD, Armstrong BG. The combination of effects on lung cancer of cigarette smoking and exposure in Quebec chrysotile miners and millers. Ann Occup Hyg. 2002;46(1): 5-13, http://dx.doi.org/10.1093/annhyg/mef008.

15. Lin S, Wang X, Yu IT, Yano E, Courtice M, Qiu H, et al. Cause-specific mortality in relation to chrysotile-asbestos exposure in a Chinese cohort. J Thorac Oncol. 2012;7(7): 1109-14, http://dx.doi.org/10.1097/JTO.0b013e3182519a60.

16. Hauptverband der Gewerblichen Berufsgenossenschaften (HVBG). [Asbostos at the workplace in the German Democratic Republic (GDR)]. Asbest an Arbeitsplätzen in der DDR. BIA-Report 3/95. Sankt Augustin; 1995 [cited 2014 Dec 5]. p. 19-20. Available from: http://publikationen.dguv.de/dguv/ udt_dguv_main.aspx?FDOCUID=24472. German.

17. Volzke H, Alte D, Schmidt CO, Radke D, Lorbeer R, Friedrich N, et al. Cohort profile: The study of health in Pomerania. Int J Epidemiol. 2010;40(2):294-307, http://dx.doi. org/10.1093/ije/dyp394.

18. Kawachi I, Kennedy BP. The relationship of income inequality to mortality: Does the choice of indicator matter. Soc Sci Med. 1997;45:1121-7, http://dx.doi.org/10.1016/ S0277-9536(97)00044-0.

19. Ganzeboom HDG, Treiman DJ. Three internationally standardised measures for comparative research on occupational status. In: Hoffmeyer-Zlotnik JHP, Wolf C, editors. Advances in cross national comparison. A European working book for demographic and socio-economic variables. New York: Kluwer Academic Press; 2003. p. 159-93, http:// dx.doi.org/10.1007/978-1-4419-9186-7_9.

20. Pira E, Pelucchi C, Piolatto PG, Negri E, Bilei T, la Vecchia C. Mortality from cancer and other causes in the Balangero cohort of chrysotile asbestos miners. Occup Environ Med. 2009;66(12):805-9, http://dx.doi.org/10.1136/ oem.2008.044693.

21. Yano E, Wang ZM, Wang XR, Wang MZ, Lan YJ. Cancer mortality among workers exposed to amphibole-free chrysotile asbestos. Am J Epidemiol. 2001;154(6):538-43, http:// dx.doi.org/10.1093/aje/154.6.538. 
22. Menegozzo S, Comba P, Ferrante D, De Santis M, Gorini G, Izzo F, et al. Mortality study in an asbestos cement factory in Naples, Italy. Ann Ist Super Sanita. 2011;47(3):296-304.

23. Wang X, Yano E, Qiu H, Yu I, Courtice MN, Tse LA, et al. A 37-year observation of mortality in Chinese chrysotile asbestos workers. Thorax. 2011;67(2):106-10, http://dx.doi. org/10.1136/thoraxjnl-2011-200169.

24. Deutsche Gesetzliche Unfallversicherung (DGUV). [Fibre years]. BK-Report 1/2013. Berlin: DGUV; 2013 [cited 2014 Dec 5]. p. 18-20. Available from: http://publikatio nen.dguv.de/dguv/udt_dguv_main.aspx?FDOCUID $=26$ 123. German.

25. Robert Koch Institut and Association of Populationbased Cancer Registries in Germany. [Cancer in Germany 2009/2010]. Berlin: Robert Koch Institut; 2013 [cited 2014 Dec 5]. p. 64-7. Available from: https://www.gbe-bund.de/pdf/ KID2013.pdf. German.

26. Neumann V, Gunthe S, Mulle KM, Fischer M. Malignant mesothelioma - German mesothelioma register 1987-1999. Int Arch Occup Environ Health. 2001;74(6):383-95, http:// dx.doi.org/10.1007/s004200100240.

27. Mossman BT. In vitro approaches for determining mechanisms of toxicity and carcinogenicity by asbestos in the gastrointestinal and respiratory tracts. Environ Health Perspect. 1983;53:155-61, http://dx.doi.org/10.1289/ehp.8 353155.

28. Clin B, Morlais F, Dubois B, Guizard AV, Desoubeaux N, Marquignon MF, et al. Occupational asbestos exposure and digestive cancers - A cohort study. Aliment Pharmacol Ther. 2009;30(4):364-74, http://dx.doi.org/10.1111/j.13652036.2009.04050.x.
29. Szeszenia-Dabrowska N, Wilczynska U, Szymczak W. Mortality of workers at two asbestos-cement plants in Poland. Int J Occup Med Environ Health. 2000;13(2):121-30.

30. Enterline PE, Hartley J, Henderson V. Asbestos and cancer: A cohort followed up to death. Br J Ind Med. 1987; 44(6):396-401, http://dx.doi.org/10.1136/oem.44.6.396.

31. Raffn E, Lynge E, Juel K, Korsgaard B. Incidence of cancer and mortality among employees in the asbestos cement industry in Denmark. Br J Ind Med. 1989;46(2):90-6, http:// dx.doi.org/10.1136/oem.46.2.90.

32. Sun TD, Chen JE, Zhang XJ, Li XY. [Cohort studies on cancer mortality of digestive system among workers exposed to asbestos: A meta-analysis]. Zhonghua Lao Dong Wei Sheng Zhi Ye Bing Za Zhi. 2008;26(10):605-8. Chinese.

33. Levin JL, McLarty JW, Hurst GA, Smith AN, Frank AL. Tyler asbestos workers: Mortality in a cohort exposed to amosite. Occup Enivron Med. 1998;55:155-60, http://dx.doi. org/10.1136/oem.55.3.155.

34. Selikhoff I, Hammond E, Seidmann H. Latency of asbestos disease among insulation workers in the United States and Canada. Cancer. 1980;46(12):2736-40.

35. Hagemeyer O, Otten H, Kraus T. Asbestos consumption, asbestos exposure and asbestos-related occupational diseases in Germany. Int Arch Occup Environ Health. 2006;79:61320, http://dx.doi.org/10.1007/s00420-006-0091-x.

36. Hauptverband der Gewerblichen Berufsgenossenschaften (HVBG). [Occupational diseases caused by asbestos - development and prognosis]. Sankt Augustin: HVBG; 2003 [cited 2014 Dec 5]. p. 31. Available from: http://publikationen. dguv.de/dguv/udt_dguv_main.aspx?FDOCUID = 24596 . German.

This work is available in Open Access model and licensed under a Creative Commons Attribution-NonCommercial 3.0 Poland License - http://creativecommons.org/ licenses/by-nc/3.0/pl/deed.en. 